\title{
1 Novel orthonairovirus in rodents and shrews, Gabon
}

3 Running Title: Novel orthonairovirus in Gabon.

5 Authors:

6 Takehiro Ozeki, Haruka Abe, Yuri Ushijima, Chiméne Nze-Nkogue, Etienne F Akomo-Okoue,

7 Ghislain W.E Ella, Lilian B.M Koumba, Branly C.B.B Nzo, Rodrigue Mintsa-Nguema, Patrice

8 Makouloutou-Nzassi, Boris K Makanga, Fred L.M Nguelet, Georgelin N Ondo, Marien J.V.M

9 Mbadinga, Yui Igasaki, Sayaka Okada, Bertrand Lell, Laura C. Bonney, Roger Hewson, Yohei

10 Kurosaki and Jiro Yasuda.

12 Author affiliations: Nagasaki University, Nagasaki, Japan (T. Ozeki, H. Abe, Y. Ushijima, Y. Igasaki, S.

13 Okada, Y. Kurosaki, J. Yasuda); L'Institut de Recherche en Ecologie Tropicale (IRET), Libreville,

14 Gabon (C. Nze-Nkogue, E.F. Akomo-Okoue, G.W.E. Ella, L.B.M. Koumba, B.C.B.B. Nzo, R.

15 Mintsa-Nguema, P. Makouloutou-Nzassi, B.K. Makanga, F.L.M. Nguelet); Centre de Recherche

16 Médicales de Lambaréné (CERMEL), Lambaréné, Gabon (G.N. Ondo, M.J.V.M. Mbadinga, B. Lell);

17 University of Tübingen, Tübingen, Germany (B. Lell); and Medical University of Vienna, Vienna,

18 Austria (B. Lell); United Kingdom Health Security Agency (UKHSA), Porton Down, Salisbury, UK

19 (L.C. Bonney, R. Hewson) 


\section{Abstract}

Small mammals harbor various zoonotic viruses and are natural reservoirs for emerging viruses. association with human neural diseases. The virus was found in $24.6 \%$ of the small mammals captured in Gabon, Central Africa.

\section{Text}

Small mammals, including rodents and shrews, are natural reservoirs of many zoonotic pathogens. These animals host highly pathogenic human viruses such as Lassa, Machupo, Junin, hanta, and Crimean-Congo hemorrhagic fever viruses, all of which belong to order Bunyavirales (1).

31 Rodent-borne viral diseases, such as Lassa fever, have emerged in sub-Saharan Africa. Thus, local

32 residents are at a potentially high risk of viral exposure under poor sanitary conditions, particularly in rural areas (2). Moreover, several viruses, including Tanganya virus of genus Orthohantavirus and Thiafora virus (TFAV) of genus Orthonairovirus, both belonging to Bunyavirales, have been identified in the musk shrews (Crocidura sp.) in Western Africa (3,4). Accordingly, small mammal populations hosting numerous zoonotic viruses may pose a public health threat to humans in sub-Saharan Africa. 
39 have been identified (5-8). In contrast, in Central Africa, no clinical cases of Lassa fever or other

40 rodent-borne human viral diseases have been reported which indicates that few small mammal-borne

41 viral surveillance studies have been conducted (5). Nonetheless, recent serological surveillance studies

42 have provided evidence of past infection of rodent-borne viruses among residents of Central African

43 countries $(9,10)$. This suggested that unrecognized zoonotic viruses might be present in this region.

44 Therefore, to understand the potential risks of transmission of known and unknown zoonotic small

45 mammal borne viruses, we investigated the viruses present among small mammal populations in

46 Gabon, Central Africa.

47

\section{The Study}

Between 2019 and 2020, 281 animals (152 rodents and 129 shrews) were captured using Sherman and Tomahawk traps placed in a forest near the suburban area, and bushes around human dwellings in

51 Lambaréné, Central Gabon. After organ specimens were collected by dissection, tissue RNA was

52 extracted from kidney homogenates, as described previously (10). Virus screening was performed

53 using the PrimeScript II High Fidelity One Step RT-PCR Kit (Takara Bio, Shiga, Japan). We initially

54 targeted partially conserved nucleotide sequences of three genera of viruses: Mammarenavirus,

55 Orthohantavirus, and Orthonairovirus, belonging to the order Bunyavirales. For the RT-PCR detection

56 of mammarenavirus and orthohantavirus, we employed the pan-viral family primer sets described

57 previously $(8,11)$. For orthonairovirus, a primer set was designed based on an alignment of sequences 
58 of a highly conserved region on the large segment (Appendix Table). All RT-PCR was performed

59 under the following conditions: $10 \mathrm{~min}$ at $45^{\circ} \mathrm{C}, 2 \mathrm{~min}$ at $94{ }^{\circ} \mathrm{C}, 35$ cycles each of $10 \mathrm{~s}$ at $98{ }^{\circ} \mathrm{C}, 15 \mathrm{~s}$ at

$6045^{\circ} \mathrm{C}$, and $10 \mathrm{~s}$ at $68{ }^{\circ} \mathrm{C}$. All amplicons were confirmed by Sanger sequencing, and the obtained

61 sequences were identified by BLAST search (https://blast.ncbi.nlm.nih.gov).

62 The species of animals captured in this study were identified by analyzing their cytochrome b

63 gene nucleotide sequences, as described previously (10). The animal species were determined by

64 BLAST search after performing Sanger sequencing.

After initial screening, viral sequences of mammarenavirus and orthohantavirus were not

66 detected in any of the samples (Table 1). By contrast, novel orthonairovirus-like sequences were

67 detected in 69 out of the 281 sampled animals (24.6\%). BLAST search revealed that the newly

68 identified sequences showed high similarity to Erve virus (ERVEV) or TFAV (62.8-74.4\%). These

69 viruses were previously identified among the Crocidura sp. found in France and Senegal, respectively

$70(4,12)$. Notably, the virus prevalence in Crocidura sp. was significantly higher than that in all other

71 captured rodents (virus prevalence in Crocidura sp. vs. rodents: 34.1\% (44/129), odds ratio: 2.63, 95\%

72 CI: $1.50-4.62, p<0.001$ ). This novel orthonairovirus was named Lamusara virus (LMSV), based on the

73 identified place (Lambaréné) and virus hosts ("musaraigne"; "shrew" in French and "ra"; "rodent" in

74 French).

75 Genus Orthonairovirus belongs to family Nairoviridae, which includes enveloped and

76 negative-sense single-stranded RNA viruses (13). Its genome comprises large, medium, and small 
segments encoding the RNA-dependent RNA polymerase (L), glycoprotein precursor (GPC), and nucleoprotein $(\mathrm{N})$, respectively (14).

$$
\text { To determine longer viral genome sequences, we performed RT-PCR using RNA samples positive }
$$

for the LMSV genome. To design the deduced primer sets, the nucleotide sequences of each genome segment of ERVEV and TFAV were aligned (GenBank accession numbers: JF911697-JF911699, KU925458-KU925460 (ERVEV), and NC_039220-NC_039222 (TFAV); Appendix Table). Once the amplicons were confirmed as the LMSV genome, Sanger sequencing was repeatedly performed to close sequence gaps.

Whole genome sequences of LMSV, consisting of three segments: large, medium, and small, were successfully determined (DDBJ accession numbers: LC671712-LC671787) in accordance with orthonairovirus genome organization. Each genome segment comprises a single open reading frame (ORF) encoding the L (11,583 nt / 3,861 aa), GPC (3,819 nt / 1,273 aa), and N (2,016 nt / 672 aa) proteins, respectively. Phylogenetic analysis was also performed among orthonairovirus protein-coding region of nucleotide sequences using IQ-TREE (http://iqtree.cibiv.univie.ac.at/) to genetically characterize LMSV. The results showed that all three LMSV encoding proteins were phylogenetically close to ERVEV and TFAV, yet formed unique phylogenetic clusters that differed from other orthonairovirus (Figure). Moreover, there were at least two distinct genotypes in the LMSV cluster of each segment according to the constructed trees. Comparison of nucleotide and amino acid sequences between two LMSV genotypes (strains CG002 vs. CG020) revealed that the sequences of 
96 the GPC protein were highly conserved $(82.5 \%$ and $92.6 \%$ at nucleotide and amino acid levels,

97 respectively), whereas the sequences of $\mathrm{L}$ and $\mathrm{N}$ proteins were relatively divergent between the two

98

99

100

101

102

103

104

105

106

107

108

109

110

111

112 113 significant sequence diversity. These results suggest the unique evolution and host adaptation of 114 LMSV circulating among wildlife in Gabon.

genotypes (L: 68.6\% and 66.9\% at nucleotide and amino acid levels, respectively; N: $59.9 \%$ and

61.4\% at nucleotide and amino acid levels, respectively) (Table 2).

LMSV showed the highest sequence similarity to TFAV compared to other orthonairovirus, at nucleotide and amino acid levels in all three ORFs (LMSV CG002 vs. TFAV; L: $66.8 \%$ and 72.0\% at nucleotide and amino acid levels, respectively; GPC: $69.0 \%$ and 70.8\% at nucleotide and amino acid levels, respectively; N: $62.3 \%$ and $62.5 \%$ at nucleotide and amino acid levels, respectively) (Table 2).

According to the criteria described by Walker et al., LMSV should be grouped into the genogroup Thiafora based on the amino acid sequence homology value ( $>52 \%)$ of the N protein between LMSV, ERVEV, and TFAV (4).

\section{Conclusions}

In this study, we identified a novel orthonairovirus, LMSV, drawn from small mammals captured in Gabon, Central Africa. According to our results, LMSV should be assigned to the genogroup Thiafora, based on its genetic relationship with ERVEV and TFAV. Further, we found that Crocidura sp. could be considered as the main host for LMSV. We also demonstrated that LMSVs have acquired 
116 diseases including thunderclap headaches (15). Here, we demonstrated preliminary evidence for the

117 presence of possible causative agents of zoonotic diseases in Gabon. However, further studies are

118 needed to understand the biological characteristics of LMSV. Moreover, surveillance studies of the

119 seroprevalence of rodent-borne viruses, such as mammarenavirus and orthohantavirus, have

120 previously been conducted among local Gabonese residents $(9,10)$. By contrast, there are no reports

121 specifically targeting seroprevalence of orthonairovirus. Taken together, our findings would provide

122 novel insight into small mammal borne virus related to public health issues in Gabon. 


\section{Acknowledgements}

125 We thank Ms. Miku Takano (Nagasaki University) and Ms. Izumi Suzumori (Japan International

Cooperation Agency (JICA)) for the management of logistics, material transportation, and linguistic

We would like to thank Editage (www.editage.com) for English language editing. This work was

131 (SATREPS) from the Japan International Cooperation Agency (JICA) and the Japan Agency for

Medical Research and Development (AMED), JP21jm0110013, AMED, JP21jm0210072, and the

\section{Author's Bio} emerging infectious diseases, virus discovery, and host innate immune defense.

\section{Address for Correspondence}

140 Jiro Yasuda, Department of Emerging Infectious Diseases, Institute of Tropical Medicine, Nagasaki

141 University, 1-12-4, Sakamoto, Nagasaki 852-8523, Japan; Tel: +81-95-819-7848; Fax:

142 +81-95-819-7851; Email: j-yasuda@nagasaki-u.ac.jp 


\section{References}

145 1. Meerburg BG, Singleton GR, and Kijlstra A. Rodent-borne diseases and their risks for public health. Crit Rev Microbiol. 2009; 35(3):221-70. https://doi.org/10.1080/10408410902989837

147

2. World

Health

Organization.

Lassa

fever.

https://www.who.int/news-room/fact-sheets/detail/lassa-fever]

3. Klempa B, Fichet-Calvet E, Lecompte E, Auste B, Aniskin V, Meisel H, et al. Novel Hantavirus

Sequences in Shrew, Guinea. Emerg Infect Dis. 2007, 13 (3), 520-522.

https://doi.org/10.3201/eid1303.061198. https://doi.org/10. 4269/ajtmh. 15-0917.

Rodents and Other Mammals in Sub-Saharan Africa. PLoS Negl Trop Dis. 2020, 14 (8), e0008589. https://doi.org/10.1371/journal.pntd.0008589. 
https://doi.org/10.1093/trstmh/trv047.

7. Klempa B, Koivogui L, Sylla O, Koulemou K, Auste B, Krüger D. H, et al. Serological Evidence of Human Hantavirus Infections in Guinea, West Africa. J Infect Dis. 2010, 201 (7), 1031-1034. https://doi.org/10.1086/651169. African Wood Mouse, Guinea. Emerg Infect Dis. 2006, 12 (5), 838-840. https://doi.org/10.3201/eid1205.051487.

9. Heinemann P, Tia M, Alabi A, Anon J.-C, Auste B, Essbauer S, et al. Human Infections by Non-Rodent-Associated Hantaviruses in Africa. J Infect Dis. 2016, 214 (10), 1507-1511. https://doi.org/10.1093/infdis/jiw401. Humans in Gabon, Central Africa. Int J Infect Dis. 2021, 105, 452-459. https://doi.org/10.3201/eid1710.10452. Sawa H. Novel Arenavirus, Zambia. Emerg Infect Dis. 2011, 17 (10), 1921-1924. probable member of Bunyaviridae family isolated from shrews (Crocidura russula) in France. 
181

182

183

184

185

186

187

188

189

190

Acta Virol. 1989;33: 270-280.

13. Garrison A. R, Alkhovsky S. V, Avšič-Županc T, Bente D. A, Bergeron É, Burt F, et al. ICTV Virus Taxonomy Profile: Nairoviridae. J Gen Virol. 2020; 101 (8), 798-799. https://doi.org/10.1099/jgv.0.001485.

14. Kuhn J. H, Wiley M. R, Rodriguez S. E, Bào Y, Prieto K, Travassos da Rosa A. P. A, et al. Genomic Characterization of the Genus Nairovirus (Family Bunyaviridae). Viruses. 2016, 8 (6). https://doi.org/10.3390/v8060164.

15. Treib J, Dobler G, Haass A, Blohn W. von, Strittmatter M, Pindur G, et al. Thunderclap Headache Caused by Erve Virus? Neurology. 1998; 50 (2), 509-511. https://doi.org/10.1212/WNL.50.2.509. 
bioRxiv preprint doi: https://doi.org/10.1101/2022.01.22.477371; this version posted January $23,2022$. The copyright holder for this preprint (which was not certified by peer review) is the author/funder. All rights reserved. No reuse allowed without permission.

Table 1. Species of captured small mammals and results of virus screening

\begin{tabular}{|c|c|c|c|c|}
\hline Mammal species & Common name & Mammarenavirus & Orthohantavirus & Orthonairovirus \\
\hline Crocidura goliath & Goliath shrew & $0 / 128^{*}$ & $0 / 128$ & $44 / 128$ \\
\hline Crocidura poensis & Fraser's musk shrew & $0 / 1$ & $0 / 1$ & $0 / 1$ \\
\hline Hybomys univittatus & Peter's striped mouse & $0 / 3$ & $0 / 3$ & $0 / 3$ \\
\hline Hylomyscus sp. & African wood mouse & $0 / 2$ & $0 / 2$ & $0 / 2$ \\
\hline Lemniscomys striatus & Typical striped grass mouse & $0 / 3$ & $0 / 3$ & $1 / 3$ \\
\hline Lophuromys sp. & Brush-furred mouse & $0 / 7$ & $0 / 7$ & $0 / 7$ \\
\hline Mus minutoides & African pygmy mouse & $0 / 41$ & $0 / 41$ & $6 / 41$ \\
\hline Mus musculus & House mouse & $0 / 3$ & $0 / 3$ & $0 / 3$ \\
\hline Oenomys hypoxanthus & Common rufous-nosed rat & $0 / 6$ & $0 / 6$ & $2 / 6$ \\
\hline Praomys misonnei & Misonne's soft-furred mouse & $0 / 65$ & $0 / 65$ & $11 / 65$ \\
\hline Rattus rattus & Black rat & $0 / 16$ & $0 / 16$ & $5 / 16$ \\
\hline \multirow[t]{2}{*}{ Stochomys longicaudatus } & Target rat & $0 / 6$ & $0 / 6$ & $0 / 6$ \\
\hline & & $0 / 281$ & $0 / 281$ & $69 / 281$ \\
\hline
\end{tabular}

192

193 Table 2. Pairwise comparisons of nucleotide and amino acid sequences among representative orthonairoviruses

\begin{tabular}{lcccccccc}
\hline & \multicolumn{7}{c}{ Nucleotide (\%) } \\
\cline { 2 - 8 } & LMSV & LMSV & ERVEV & TFAV & CCHFV & HAZV & NSDV & DUGV \\
\hline L & CG020 & & & & & & \\
LMSV CG002 & & 68.6 & 65.0 & 66.8 & 53.5 & 52.0 & 52.7 & 54.1 \\
LMSV CG020 & 66.9 & & 67.1 & 68.4 & 53.0 & 53.1 & 53.3 & 55.3 \\
ERVEV & 69.6 & 67.9 & & 68.8 & 52.4 & 51.3 & 51.5 & 53.1 \\
TFAV & 72.0 & 69.0 & 73.9 & & 52.2 & 51.2 & 52.3 & 52.6 \\
CCHFV & 47.7 & 45.6 & 47.9 & 49.1 & & 63.4 & 64.5 & 62.9 \\
HAZV & 48.7 & 47.4 & 49.7 & 49.9 & 66.8 & & 65.4 & 63.8 \\
NSDV & 48.1 & 46.7 & 48.9 & 50.8 & 67.5 & 71.2 & & 66.3 \\
DUGV & 48.7 & 47.2 & 48.7 & 49.8 & 65.2 & 67.8 & 70.2 & \\
\hline GPC & & & & & & & & \\
LMSV CG002 & & 82.5 & 67.9 & 69.0 & 43.8 & 44.8 & 43.5 & 45.3 \\
LMSV CG020 & 92.6 & & 66.7 & 67.7 & 43.6 & 44.6 & 43.4 & 45.1 \\
ERVEV & 68.5 & 68.3 & & 70.0 & 43.5 & 44.9 & 45.0 & 45.1 \\
TFAV & 70.8 & 70.4 & 73.0 & & 42.9 & 44.2 & 43.1 & 44.7 \\
CCHFV & 35.1 & 35.4 & 37.4 & 35.6 & & 49.7 & 53.6 & 53.4 \\
HAZV & 39.5 & 39.2 & 39.7 & 39.5 & 41.6 & & 59.1 & 54.7 \\
NSDV & 37.2 & 37.2 & 37.6 & 37.2 & 45.8 & 44.4 & & 58.8 \\
DUGV & 37.0 & 36.9 & 37.2 & 35.9 & 44.1 & 50.4 & 55.6 & \\
\hline N & & & & & & & & \\
LMSV CG002 & & 59.9 & 60.9 & 62.3 & 53.3 & 52.9 & 54.7 & 40.6 \\
LMSV CG020 & 61.4 & & 68.9 & 70.9 & 52.4 & 51.2 & 52.3 & 51.4 \\
ERVEV & 61.2 & 72.9 & & 67.9 & 51.7 & 51.2 & 51.5 & 51.4 \\
TFAV & 62.5 & 76.2 & 60.8 & & 52.6 & 50.2 & 51.7 & 51.7 \\
CCHFV & 45.5 & 42.9 & 42.2 & 44.9 & & 61.1 & 63.1 & 60.4 \\
HAZV & 44.7 & 41.6 & 42.1 & 42.7 & 60.3 & & 63.2 & 59.9 \\
NSDV & 46.0 & 43.1 & 43.8 & 45.3 & 62.2 & 64.0 & & 63.5 \\
DUGV & 41.8 & 42.3 & 44.2 & 44.2 & 57.7 & 56.2 & 60.5 & \\
\cline { 2 - 9 } & & & & Amino acid(\%) & & & \\
\hline
\end{tabular}


bioRxiv preprint doi: https://doi.org/10.1101/2022.01.22.477371; this version posted January 23, 2022. The copyright holder for this preprint (which was not certified by peer review) is the author/funder. All rights reserved. No reuse allowed without permission.

Partial sequence of L protein at position 6,913-11,471 nt on LMSV L-segment (DDBJ accession numbers
LC671712 for CG002 and LC671780 for CG020) and complete nucleotide and amino acid sequences of
GPC and N protein were analyzed by MEGA7 software (https://www.megasoftware.net/). Percent values of
nucleotide and amino acid sequence identities are indicated in the upper right half and lower left half,
respectively. LMSV, Lamusara virus (DDBJ accession numbers: LC671712-LC671714 for CG002 and
LC671780-LC671782 for CG020); ERVEV, Erve virus (GenBank accession numbers: JF911697-JF911699);
TFAV, Thiafora virus (GenBank: NC_039220-NC_039222); CCHFV, Crimean-Congo hemorrhagic fever
virus (GenBank: NC_005300-NC_005302); HAZV, Hazara virus (GenBank: NC_038709-NC_038711);
NSDV, Nairobi sheep disease virus (GenBank: EU697950, EU697951 and AF504294); DUGV, Dugbe virus
(GenBank: NC_004157-NC_004159).

\begin{tabular}{lll}
\hline Primer name & Description & Sequence (5'-3') \\
\hline Primers used for virus screening & CACATAGTTGGGCCCCACTTGCTGTGATC \\
Arena-F* & Forward primer for mammarenavirus detection & AGGATAAGTGAAAGAGAGAGTAATTC \\
Arena-R* & Reverse primer for mammarenavirus detection & TGCWGATGCHACIAARTGGTC \\
HAN-L-F2* & Forward primer for orthohantavirus detection & GCRTCRTCWGARTGRTGDGCAA \\
HAN-L-R2* & Reverse primer for orthohantavirus detection & AARTGGGGYCCIATICACTGYTGYTC \\
Nairo-F & Forward primer for orthonairovirus detection & GGRACACTRTTRTACATISCYTGTTG \\
Nairo-R & Reverse primer for orthonairovirus detection & \\
Primers used for amplification of Lamusara virus genome & CGAGTATCTCAAAGAAAGCAATCCC \\
LMSV-L-F1 & Forward primer for L protein at position 1-4,809 nt & CTCCCAGTTCATATGTCTMTCTGCAG \\
LMSV-L-R1 & Reverse primer for L protein at position 1-4,809 nt & AATGGCATAAGCAGATTRTCCTG \\
LMSV-L-F2 & Forward primer for L protein at position $4,390-7,013 \mathrm{nt}$ & GCAGGRATTTCAATTTGTCGAC \\
LMSV-L-R2 & Reverse primer for L protein at position $4,390-7,013 \mathrm{nt}$ & TAACTCATGCTGGCAGTTCAGATG \\
LMSV-L-F3 & Forward primer for L protein at position 7,404-11,583 nt & TTTCTCAAAGATAGTATTCCCCCCTACC \\
LMSV-L-R3 & Reverse primer for L protein at position 7,404-11,583 nt & TCTCAAAGAAAGWCTAGCGGCAAACTCGTC \\
LMSV-M-F1 & Forward primer for GPC at position 1-3,011 nt & CCAGCTTCCTGTGGTGCAGTAGTAGTC \\
LMSV-M-R1 & Reverse primer for GPC at position 1-3,011 nt & ACTTGGTGYTGGGGAGTTGGWACAG \\
LMSV-M-F2 & Forward primer for GPC at position 2,505-3,810 nt & TCTCAAAGATATAGGAGCGGCATACTCG \\
LMSV-M-R2 & Reverse primer for GPC at position 2,505-3,810 nt & TCTCAAAGAAAGYGTGCTGYATACTG \\
LMSV-S-F & Forward primer for N protein at position 1-2,016 nt & TCTCAGAAGATAGTGTTGCTGCATACTG \\
LMSV-S-R & Reverse primer for N protein at position 1-2,016 nt & \\
\hline${ }^{*}$ Published primers (8,11) &
\end{tabular}

198 Maximum-likelihood phylogenetic trees of LMSV were constructed based on L protein partial

199 nucleotide sequences (at position 6,913-11,471 nt on LMSV large-segment (DDBJ accession number:

200 LC671712)) (A), GPC complete nucleotide sequences (B) and N protein complete nucleotide

201 sequences (C) using IQ-tree (http://iqtree.cibiv.univie.ac.at/) with 1,000 bootstraps. Bootstrap values

202 of $\geqq 70 \%$ are shown at the nodes of the trees. Nine genogroups of genus Orthonairovirus are shown on 
bioRxiv preprint doi: https://doi.org/10.1101/2022.01.22.477371; this version posted January 23, 2022. The copyright holder for this preprint (which was not certified by peer review) is the author/funder. All rights reserved. No reuse allowed without permission.

203 the right of the trees (4) and LMSV clusters in each segment were circled by broken line. The scale bar

204 indicates nucleotide substitutions per site. 


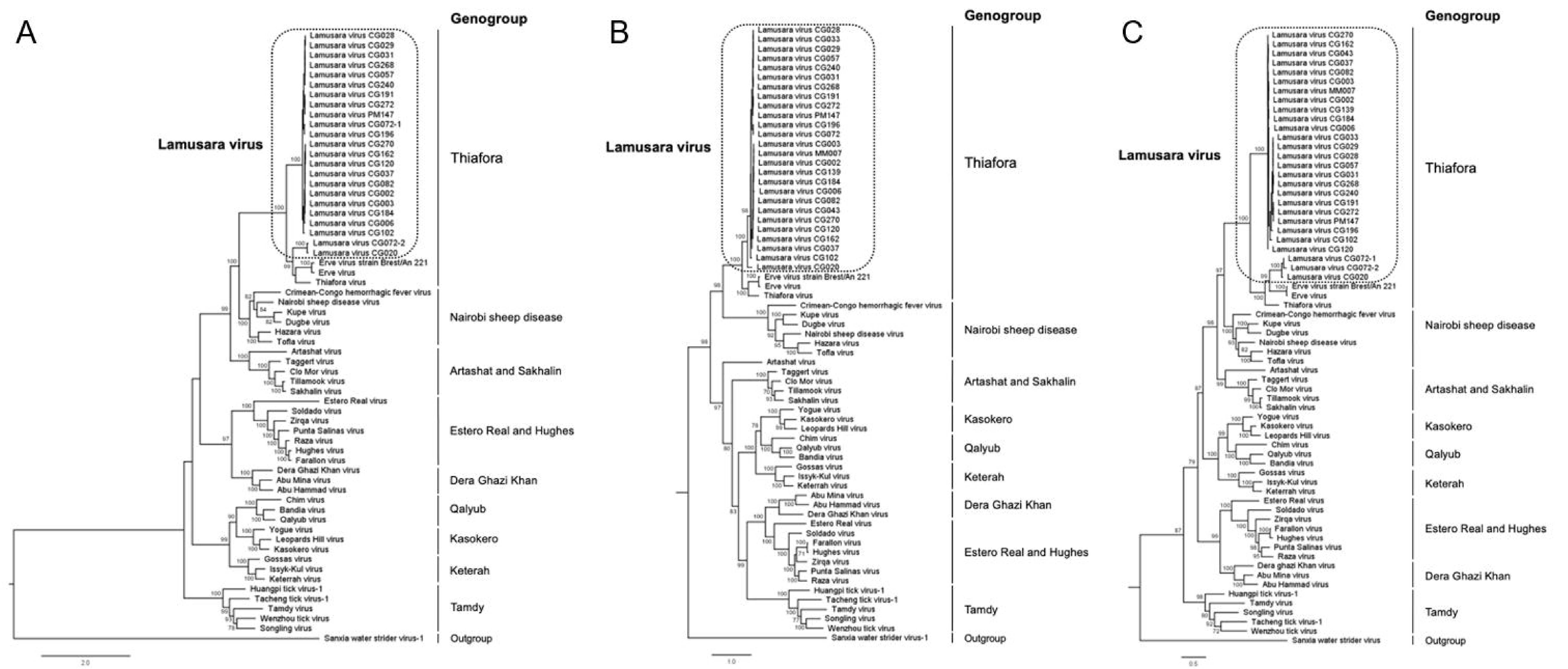

